https://doi.org/10.22319/rmcp.v12i2.5480

Nota de investigación

\title{
Eficacia del timol en el control del hongo Nosema ceranae infectando abejas africanizadas
}

Azucena Vargas-Valero ${ }^{a}$

Roberto C. Barrientos-Medina ${ }^{b}$

Luis A. Medina Medina ${ }^{\mathrm{c} *}$

a Instituto Nacional de Investigaciones Forestales, Agrícolas y Pecuarias. Centro de Investigación Regional del Sureste, Campo Experimental Edzná, Campeche, México.

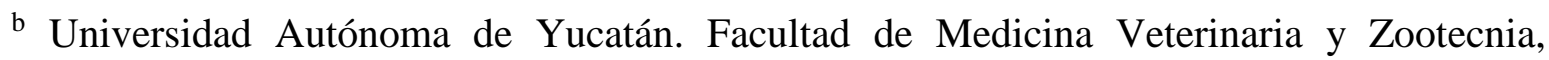
Departamento de Ecología, Yucatán, México.

c Universidad Autónoma de Yucatán. Facultad de Medicina Veterinaria y Zootecnia, Departamento de Apicultura, Yucatán, México.

* Autor de correspondencia: mmedina@ correo.uady.mx

\section{Resumen:}

Nosema ceranae, es un parásito obligatorio del intestino medio de las abejas melíferas que causa destrucción de las células epiteliales afectando la digestión y asimilación del alimento, impactando negativamente el desarrollo y sobrevivencia de las colonias de abejas. Con la finalidad de reducir los efectos negativos, se evaluó la eficacia del timol en el control de esta parasitosis. El estudio se realizó en dos apiarios experimentales con un total de 56 colonias de abejas distribuidas en tres grupos experimentales: G1) 18 colonias que recibieron tratamiento con fumagilina como producto de referencia ( $25.2 \mathrm{mg}$ de fumagilina/semana); G2) 19 colonias que recibieron tratamiento con timol como producto alternativo (66 mg de cristales/semana) y G3) 19 colonias que no recibieron ningún tratamiento (grupo testigo). Los tratamientos con la fumagilina y timol se aplicaron a través del jarabe de azúcar una vez por semana durante cuatro semanas consecutivas. Los niveles de infección de $N$. ceranae se estimaron en el macerado del abdomen de 60 abejas adultas colectadas de cada colonia 
experimental. Los resultados al final de los tratamientos indican que las colonias que recibieron fumagilina (G1) disminuyeron sus niveles de infección de 123,529 a 1,805 esporas por abeja; para G2 (timol), la reducción fue de 133,438 a 28,099 esporas por abeja, y para las colonias del grupo testigo (G3) fue de 119,306 a 36,447 esporas por abeja. Los tres grupos experimentales presentaron diferencias estadísticas significativas en los niveles de infección de $N$. ceranae al final de los tratamientos. La fumagilina presentó una mayor eficacia (95.2\%) en comparación con el timol la, cual fue baja (31.1\% de eficacia) indicando que se requieren estudios adicionales para determinar la concentración más efectiva a nivel de colonia bajo condiciones tropicales de Yucatán, a fin de que este aceite esencial de origen vegetal sea incorporado como producto alternativo para el control de esta parasitosis.

Palabras clave: Nosema ceranae, Nosemosis, Fumagilina, Timol, Eficacia, Apis mellifera.

Recibido: 20/08/2019

Aceptado: 02/09/2020

La nosemosis, es una parasitosis que afecta el tracto digestivo de las abejas Apis mellifera y es causado por dos especies de hongos de la familia Nosematidae: Nosema apis, especie originalmente asociada a esta infección en las abejas melíferas y Nosema ceranae, especie originalmente asociada a la abeja asiática Apis cerana y diagnosticada por primera vez infectando a las abejas A. mellifera en el centro y norte de España en $2006^{(1)}$ donde se había registrado durante el invierno una reducción en la producción de miel y una alta mortalidad de colonias de abejas ${ }^{(2,3)}$.

Ambas especies de microsporidios (N. apis y N. ceranae) se reproducen rápidamente dentro de las células epiteliales del intestino medio (ventrículo) de las abejas adultas (reina, obreras y zánganos) ocasionando la destrucción de las células epiteliales encargadas de la digestión y asimilación del alimento ${ }^{(3)}$ lo que ocasiona un estrés nutricional en las abejas infectadas ${ }^{(4)}$. Otros daños observados a nivel individual demuestran una reducción en el periodo de vida ${ }^{(3,5,6)}$ y un efecto negativo en la orientación y habilidades como forrajeadoras en las obreras infectadas $^{(7,8)}$.

A nivel de colonia, los daños causados por la nosemosis pueden ser graves cuando la prevalencia y niveles de infección son altos, ocasionando una reducción en las áreas de cría y en la población de abejas adultas y en consecuencia una reducción en la producción de miel de las colonias ${ }^{(9,10)}$ hasta llegar al colapso y pérdida de las mismas ${ }^{(11,12)}$. 
A partir de 2006 cuando se diagnosticó la infección en las abejas A. mellifera, la especie $N$. ceranae se ha convertido en uno de los patógenos más ampliamente distribuidos a nivel mundial $^{(13,14)}$, y ha estado asociada a una alta mortalidad de colonias de abejas en Europa ${ }^{(11)}$, Norteamérica $^{(15)}$ y Sudamérica ${ }^{(16)}$ presentando una mayor virulencia en comparación con la infección causada por $N$. apis ${ }^{(5,17,18)}$.

En Yucatán, los apicultores no han reportado la pérdida masiva o alta mortalidad de colonias de abejas debido a la infección por $N$. apis o $N$. ceranae. Sin embargo, a partir de $2008^{(19)}$ se ha reportado una elevada prevalencia de la nosemosis ( 74 a $100 \%$ ) en colonias provenientes de apiarios comerciales en comparación con lo reportado en años anteriores $(7.2 \%)^{(20)}$, y esta alta prevalencia se ha atribuido a la presencia de la especie $N$. ceranae, infectando a las abejas africanizadas en Yucatán ${ }^{(21)}$. Recientemente, se confirmó que la infección causada por $N$. ceranae afecta negativamente el inicio y duración de forrajeo en abejas de origen africanizado, así como la longevidad de las obreras bajo las condiciones tropicales de Yucatán $^{(22)}$.

Para el control de la nosemosis, el único medicamento considerado eficaz contra este microsporidio ha sido la fumagilina (sal de diciclohexilamonio), un antibiótico obtenido del hongo Aspergillus fumigatus y que históricamente se ha utilizado para controlar esta parasitosis causada por $N$. apis en abejas melíferas desde que fue evaluado a principios de la década de los $50^{(23)}$. El mecanismo de acción de la fumagilina, es la inhibición de la replicación del ADN del microsporidio suprimiendo su reproducción, lo que resulta en una disminución del número de esporas en el ventrículo de las abejas ${ }^{(24,25)}$.

A diferencia de los Estados Unidos de América donde las infecciones causadas por $N$. apis o $N$. ceranae pueden ser controladas con la aplicación de fumagilina, en diversos países de Europa $^{(26)}$ así como en México, este antibiótico no está autorizado para el control de la nosemosis debido a la toxicidad que puede representar para los humanos, por lo que cualquier residuo que quede en la miel de las colonias que se encuentren bajo tratamiento, representa un riesgo directo para el consumidor ${ }^{(27)}$.

Debido a estos inconvenientes, algunos productos alternativos han sido propuestos para el control de esta parasitosis, incluyendo diferentes aceites esenciales de origen vegetal como el timol obtenido del tomillo (Thymus vulgaris), el aceite esencial de vetiver (Chrysopogon zizanioides) así como el resveratrol, que es un polifenol natural presente en numerosas plantas y frutos como la uva. Estos aceites esenciales han presentado resultados favorables en el control de la nosemosis, principalmente el timol ${ }^{(28,29)}$. Se ha demostrado que colonias con nosemosis que fueron tratadas con timol aplicado a través de un jarabe de azúcar en una concentración de $0.44 \mathrm{mM}$, presentaron una reducción en el número de esporas por abeja y registraron un incremento en las áreas de cría, población de abejas adultas y producción de 
miel posterior a la aplicación de este producto en comparación con las colonias de abejas que no recibieron ningún tipo de tratamiento ${ }^{(28)}$. Adicionalmente, se considera que por su naturaleza, los productos alternativos a base de aceites esenciales no representan toxicidad para las abejas, presentan un menor riesgo de dejar residuos en la miel ${ }^{(30)}$ y son de menor costo para el apicultor.

Debido a la necesidad de contar con productos alternativos, el objetivo del presente trabajo fue comparar la eficacia del timol en el control de la nosemosis causada por la especie $N$. ceranae infectando abejas africanizadas (A. mellifera) bajo condiciones tropicales de Yucatán.

El experimento se realizó en dos apiarios pertenecientes a la Facultad de Medicina Veterinaria y Zootecnia de la Universidad Autónoma de Yucatán (UADY) ubicados en la

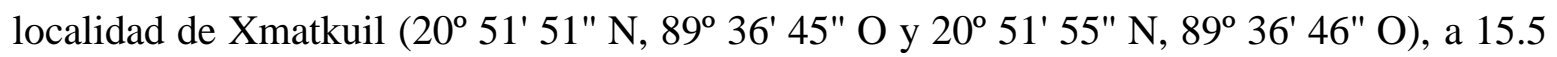
km de la ciudad de Mérida, Yucatán. Esta región presenta un clima cálido sub-húmedo con lluvias en verano (Awo), con una precipitación pluvial promedio anual de $985 \mathrm{~mm}$, temperatura promedio anual de $26.8^{\circ} \mathrm{C}$ y humedad relativa promedio anual de $78 \%(31)$.

Las colonias de abejas en ambos apiarios eran colonias dobles (cámara de cría y un alza) alojadas en colmenas tipo Langstroth, que contaban con reinas africanizadas fecundadas naturalmente, con una población de abejas adultas que cubrían entre 7 a 9 panales de la cámara de cría y con 6 a 8 panales conteniendo cría en diferentes etapas (huevos, larvas y pupas), además de contar con panales con miel y polen. Las colonias fueron distribuidas de manera similar entre los grupos experimentales y todas se encontraban infectadas con la especie $N$. ceranae, la cual fue identificada a través de la técnica de PCR punto final ${ }^{(32)}$ en muestras de abejas forrajeadoras colectadas en la entrada de cada colonia experimental.

Antes de iniciar las evaluaciones, se realizó un diagnóstico preliminar en ambos apiarios para determinar el nivel de infección de $N$. ceranae (número de esporas/abeja) en todas las colonias de abejas, con la finalidad de que los tres grupos experimentales presenten un nivel de infección similar al inicio del experimento. Para determinar el nivel de infección, se colectaron muestras de abejas adultas ( 100 a 150 obreras) de la entrada de cada colonia experimental. Sesenta abejas de cada muestra fueron analizadas para determinar la presencia de esporas de $N$. ceranae y se cuantificó la severidad de la infección (número de esporas/abeja) a través del conteo de esporas presentes en el tracto digestivo de las obreras, la cual consiste en retirar el abdomen de las 60 abejas adultas y colocarlas en un mortero añadiendo $60 \mathrm{ml}$ de agua destilada ${ }^{(9,33)}$. Los abdómenes fueron macerados hasta obtener una mezcla homogénea y la suspensión se filtró a través de una gasa para eliminar impurezas. Una gota de la solución fue colocada en ambos retículos de una cámara de Neubauer donde 
se observó a 400 aumentos y se registró el número de esporas del macerado, determinando el nivel de infección (número de esporas) promedio por abeja.

La evaluación de la eficacia de la fumagilina (Fumagilin-B ${ }^{\circledR}$ ) y los cristales de timol (SigmaAldrich; $\geq 99.5 \%$ de pureza) en el control de $N$. ceranae se realizó durante un período de cuatro semanas y las colonias de abejas de ambos apiarios se dividieron en tres grupos experimentales, los cuales fueron:

Grupo 1 (G1): con 18 colonias de abejas que recibieron tratamiento con fumagilina como producto de referencia (Fumagilin- ${ }^{\circledR}$ ), aplicando $1.2 \mathrm{~g}$ del producto comercial $(25.2 \mathrm{mg}$ de fumagilina base) por colonia/semana en un litro de jarabe de azúcar (2:1, azúcar: agua); Grupo 2 (G2): con 19 colonias que recibieron tratamiento con timol como producto alternativo, aplicando $66 \mathrm{mg}$ de cristales de timol (99.5\% de pureza) por colonia/semana en un litro de jarabe de azúcar (2:1, azúcar: agua), y el Grupo 3 (G3): con 19 colonias de abejas a las cuales se les suministró únicamente un litro de jarabe de azúcar (2:1, azúcar: agua) por colonia/semana, sin ningún tipo de tratamiento, conformando el grupo testigo. Los tratamientos se aplicaron una vez por semana durante cuatro semanas consecutivas.

Al final de cada semana de aplicación, abejas adultas se colectaron de la entrada de cada colonia con la finalidad de determinar el nivel de infección para cada grupo experimental, de acuerdo con la metodología descrita con anterioridad.

Los datos originales (número de esporas por abeja) fueron divididos entre mil, para facilitar su manejo estadístico, y se utilizó la transformación Box-Cox ${ }^{(34)}$ para normalizar la distribución de la variable de interés. Finalmente, se empleó un análisis de varianza de medidas repetidas para comparar los promedios por tratamiento (grupos experimentales), semana y la interacción de ambos factores (tratamiento x semana). El nivel de significancia empleado fue del 5\% y los cálculos se realizaron con el programa PAST versión 3.20 ${ }^{(35)}$.

Antes de iniciar la aplicación de los tratamientos, se observó que los niveles de infección de $N$. ceranae en los tres grupos experimentales, no presentaron diferencias estadísticas significativas de acuerdo con la prueba de Kruskal-Wallis $(\mathrm{H}=0.5587, P=0.7563)$, indicando una distribución similar de los niveles de infección de las colonias para los tres grupos experimentales (Cuadro 1). 
Cuadro 1: Número de esporas por abeja $(\bar{X} \pm \mathrm{EE})$ registrado en los tres grupos experimentales después de la aplicación de cada tratamiento para el control de Nosema ceranae

\begin{tabular}{|c|c|c|c|c|}
\hline & Fumagilina & Timol & Testigo & $\mathbf{H}$ \\
\hline Día 0 & $123,529 \pm 41,220^{\mathrm{a}}$ & $133,438 \pm 59,291^{\mathrm{a}}$ & $119,306 \pm 53,100^{\mathrm{a}}$ & 0.5587 \\
\hline $\begin{array}{l}\text { Día } 7 \\
\left(1^{\text {ra }} \text { aplicación }\right)\end{array}$ & $104,688 \pm 42,293^{\mathrm{a}}$ & $67,812 \pm 14,426^{\mathrm{a}}$ & $80,394 \pm 22,381^{a}$ & 1.8960 \\
\hline $\begin{array}{l}\text { Día } 14 \\
\left.\text { (2 }{ }^{\mathrm{da}} \text { aplicación }\right)\end{array}$ & $57,666 \pm 14,827^{\text {a }}$ & $84,500 \pm 23,054^{\mathrm{a}}$ & $96,666 \pm 25,476^{\mathrm{a}}$ & 2.1100 \\
\hline $\begin{array}{l}\text { Día } 21 \\
\left.\text { ( } 3^{\text {ra }} \text { aplicación }\right)\end{array}$ & $30,468 \pm 11,190^{\text {a }}$ & $27,058 \pm 9,779^{a}$ & $42,631 \pm 9,055^{\mathrm{a}}$ & 4.9040 \\
\hline $\begin{array}{l}\text { Día } 28 \\
\left(4^{\text {ta }} \text { aplicación }\right)\end{array}$ & $1,805 \pm 527^{\mathrm{a}}$ & $28,099 \pm 17,574^{b}$ & $36,447 \pm 17,554^{c}$ & 24.0900 \\
\hline Eficacia final, $\%$ & 95.2 & 31.1 & - & \\
\hline
\end{tabular}

De acuerdo con los resultados obtenidos, se observó que las colonias que recibieron tratamiento con la fumagilina (Fumagilin- ${ }^{\circledR}$ ), presentaron una disminución de los niveles de infección $(\bar{X} \pm$ E.E. $)$ de $123,529 \pm 41,200$ a 1,805 \pm 527 esporas por abeja al final de las cuatro semanas de tratamiento, lo que representa una eficacia del $95.2 \%$ de este antibiótico en el control de $N$. ceranae (Cuadro 1). Para las colonias del grupo que recibieron el jarabe con los cristales de timol (G2), el número de esporas se redujo de 133,438 $\pm 59,291$ a 28,099 $\pm 17,574$ esporas por abeja, representando una eficacia del $31.1 \%$. La disminución natural de los niveles de infección de la nosemosis observada en el grupo testigo, puede ser resultado de las fluctuaciones estacionales que se observan durante los diferentes meses del año, las cuales como se ha reportado en otros trabajos ${ }^{(36)}$, pueden variar drásticamente entre las diferentes temporadas del ciclo apícola.

Durante las tres primeras semanas de aplicación de los tratamientos, el número de esporas por abeja registrado en los tres grupos experimentales no presentaron diferencias estadísticas significativas, las cuales fueron significativas hasta al final de las cuatro semanas de tratamiento (Cuadro 1) donde el grupo que recibió la fumagilina, presentó una mayor eficacia seguido por el grupo que recibió el timol. La diferencia en la reducción de la infección registrada hasta el final de la cuarta semana del grupo que recibió la fumagilina (G1), indica que este antibiótico alcanza su mayor eficacia cuando las colonias de abejas recibieron los $100.8 \mathrm{mg}$ de fumagilina base ( $4.8 \mathrm{~g}$ de Fumagilin- $\mathrm{B}^{\circledR}$ en total) de acuerdo con las indicaciones del laboratorio fabricante. 
Los resultados obtenidos en este trabajo indican que la aplicación de la fumagilina disminuyó significativamente los niveles de infección de $N$. ceranae hasta la cuarta semana de aplicación y fue diferente a los niveles de infección registrados en las colonias que recibieron timol (G2) o solo recibieron el jarabe de azúcar sin tratamiento (G3), lo cual coincide con lo reportado por diversos autores en diferentes países ${ }^{(37-40)}$, donde señalan que este antibiótico continúa siendo apropiado para controlar la infección causada por la especie $N$. ceranae. Sin embargo, aun cuando este antibiótico es eficiente en reducir temporalmente los niveles de infección de $N$. ceranae, no evita las reinfecciones en las colonias después de seis meses de aplicados los tratamientos ${ }^{(38)}$.

La eficacia del timol registrada en este trabajo (31.1\%), fue inferior a lo observado bajo condiciones de laboratorio en abejas infectadas por $N$. ceranae que fueron alimentadas con jarabe de azúcar conteniendo timol, donde se observó una reducción del $40 \%$ del nivel de infección en comparación al grupo testigo que no recibió ningún tratamiento para el control de esta parasitosis ${ }^{(41)}$.

Se reporta que el timol presenta una mayor eficacia en el control de la nosemosis a partir del tercer año de la aplicación del producto ${ }^{(28)}$ a diferencia del presente trabajo donde este aceite esencial de origen vegetal fue aplicado en una sola temporada, por lo que sería recomendable seguir aplicando el producto durante dos años adicionales para verificar la eficacia de la dosis suministrada.

De acuerdo con los resultados obtenidos en este trabajo, se puede concluir que la fumagilina demuestra eficacia en reducir los niveles de infección de la nosemosis causada por la especie $N$. ceranae infectando abejas africanizadas bajo condiciones de clima tropical. Sin embargo, la aplicación de este antibiótico no está autorizado en México para uso en abejas y se recomienda su aplicación cuando los niveles de infección sean superiores a los dos millones de esporas/abeja y se respeten las dosis suministradas ${ }^{(28)}$. En este sentido y considerando que los niveles de infección de $N$. ceranae en este trabajo fueron inferiores a este valor (dos millones de esporas/abeja) antes de iniciar los tratamientos, es posible que la aplicación del timol reduzca los niveles de infección de $N$. ceranae en abejas africanizadas establecidas bajo condiciones tropicales de Yucatán.

Sin embargo, para que el timol sea considerado en el control alternativo de este hongo, se requiere realizar otras evaluaciones para tener resultados favorables en su eficacia, ya sea incrementando la dosis aplicada en cada tratamiento, el número de aplicaciones y el período o época de aplicación, para que pueda ser utilizado como una alternativa de control, ya que por su origen presenta un menor riesgo de dejar residuos en la miel. 


\section{Literatura citada:}

1. Higes M, Martin R, Meana A. Nosema ceranae, a new microsporidian parasite in honeybees in Europe. J Inv Pathol 2006;(92):81-83.

2. Higes M, Martín R, Sanz A, Álvarez N, Sanz A, García MP, Meana A. El síndrome de despoblamiento de las colmenas en España. Consideraciones sobre su origen. Vida Apícola 2005;(133):15-21.

3. Higes M, Garcia-Palencia P, Martin-Hernandez R, Meana A. Experimental infection of Apis mellifera honeybees with Nosema ceranae (Microsporidia). J Invertebr Pathol 2007;(94):211-217.

4. Mayack C, Naug D. Energetic stress in the honeybee Apis mellifera from Nosema ceranae infection. J Inv Pathol 2009;(100):185-188.

5. Paxton RJ, Klee J, Korpela S, Fries I. Nosema ceranae has infected Apis mellifera in Europe since at least 1998 and may be more virulent than Nosema apis. Apidologie 2007;(38):558-565.

6. Goblirsch M, Huang ZY, Spivak M. Physiological and behavioral changes in honey bees (Apis mellifera) induced by Nosema ceranae infection. PLos One 2013;8(3):e58165.

7. Kralj J, Fuchs S. Nosema sp. influences flight behavior of infected honey bee (Apis mellifera) foragers. Apidologie 2010;(41):21-28.

8. Wolf S, McMahon DP, Lim KS, Pull CD, Clark SJ, Paxton RJ, Osborne JL. So near and yet so far: harmonic radar reveals reduced homing ability of Nosema infected honeybees. PLoS One 2014;9(8):e103989.

9. Fries I, Ekbohm G, Villumstad E. Nosema apis, sampling techniques and honey yield. J Apic Res 1984;(23):102-105.

10. Botías C, Martin-Hernandez R, Barrios L, Meana A, Higes M. Nosema spp. infection and its negative effects on honey bees (Apis mellifera iberiensis) at the colony level. Vet Res 2013;(44):25.

11. Higes M, Martín-Hernandez R, Botias C, Bailon EG, Gonzales-Porto A, Barrios L, del Nozal MJ, Palencia PG, Meana A. How natural infection by Nosema ceranae causes honeybee colony collapse. Environ Microbiol 2008;10(10):2659-2669.

12. Higes M, Martin-Hernandez R, Garrido-Bailon E, Gonzalez-Porto AV, Garcia-Palencia $\mathrm{P}$, Meana A, et al. Honeybee colony collapse due to Nosema ceranae in professional apiaries. Environ Microbiol Rep 2009;1(2):110-113. 
13. Klee J, Besana AM, Genersch E, Gisder S, Nanetti A, Tam DO, et al. Widespread dispersal of the microsporidian Nosema ceranae, an emergent pathogen of the western honeybee, Apis mellifera. J Invertebr Pathol 2007;96(1):1-10.

14. Goulson D, Nicholls E, Botías C, Rotheray EL. Bee declines driven by combined stress from parasites, pesticides, and lack of flowers. Science 2015;347(6229):1255957.

15. vanEngelsdorp D, Evans JD, Saegerman C, Mullin C, Haubruge E, Nguyen BK, et al. Colony collapse disorder: A descriptive study. PLoS One 2009;4(8):e6481.

16. Martínez J, Leal G, Conget P. Nosema ceranae an emergent pathogen of Apis mellifera in Chile. Parasitol Res 2012;(111):601-607.

17. Fries I. Nosema apis - a parasite in the honey bee colony. Bee World 1993;(74): 5-19.

18. Webster TC, Pomper KW, Hunt G, Thacker EM. Nosema apis infection in worker and queen Apis mellifera. Apidologie 2004;(35):49-54.

19. Martinez-Puc JFM, Medina, LAM, Catzin-Ventura GA. Frecuencia de Varroa destructor, Nosema apis y Acarapis woodi en colonias manejadas y enjambres silvestres de abejas (Apis mellifera) en Mérida, Yucatán, México. Rev Mex Cienc Pecu 2011;2(1):25-38.

20. García-Millán M, Quezada-Euan JJG. Distribución de Nosemiasis en apiarios comerciales del estado de Yucatán. Apicultura Moderna 1993;(5): 22-24.

21. Briceño-Uc AR. Estudio retrospectivo de microsporidos (Nosema apis y Nosema ceranae) en colmenas de Apis mellifera de Yucatán, México. [tesis maestría]. Mérida, Yucatán. Universidad Autónoma de Yucatán. 2013.

22. Fleites-Ayil FA, Quezada-Euan JJG, Medina ML. Onset of foraging and lifespan of Africanized honey bees (Apis mellifera) infected with different levels of Nosema ceranae spores in Neotropical Mexico. Apidologie 2018;(49):781-788.

23. Katznelson H, Jamieson CA. Control of Nosema disease of honey bees with fumagillin. Science 1952;(115):70-71.

24. Hartwig A, Przelecka A. Nucleic acids in the intestine of Apis mellifera infected with Nosema apis and treated with Fumagillin DCH: cytochemical and autoradiographic studies. J Invertebr Pathol 1971;18(3):331-336.

25. Liu TP. Ultrastructural changes in the secretion granules of the hypopharyngeal glands of the honeybee infected by Nosema apis and after treatment with fumagillin. Tissue Cell 1990;22(4):523-531. 
26. Fries I. Nosema ceranae in European honey bees (Apis mellifera). J Invertebr Pathol 2010;(103):S73-S79.

27. van den Heever JP, Thompson TS, Curtis JM, Ibrahim A, Pernal SF. Fumagillin: an overview of recent scientific advances and their significance for apiculture. J Agric Food Chem 2014;(62):2728-2737.

28. Yücel B, Doğaroğlu M. The impact of Nosema apis Z. infestation of honey bee (Apis mellifera L.) colonies after using different treatment methods and their effects on the population levels of workers and honey production on consecutive years. Pakistan J Biol Sci 2005;8(8):1142-1145.

29. Maistrello L, Lodesani M, Costa C, Leonardi F, Marani G, Caldon M, Mutinelli F, Granato A. Screening of natural compounds for the control of nosema disease in honeybees (Apis mellifera). Apidologie 2008;(39):436-445.

30. Bogdanov S, Imdorf A, Kilchenmann V. Thymol residues in wax and honey after Apilife VAR Treatment. Swiss Bee Research Centre 1998;(133):1-9.

31. Orellana LR, Espadas MC, Nava MF. Climas. In: Durán R, Méndez M. editores. Biodiversidad y desarrollo humano en Yucatán. Yucatán, México: CONABIOSEDUMA; 2010:10-11.

32. Chen Y, Evans J, Smith B, Pettis J. Nosema ceranae is a long-present and wide-spread microsporidian infection of the European honey bee (Apis mellifera) in the United States. J Invertebr Pathol 2007;(97):186-188.

33. Cantwell GE. Standard methods for counting Nosema spores. Am Bee J 1970;110(6):222223.

34. Krebs CJ. Ecological Methodology. 2nd ed. Menlo Park, California, USA: AddisonWelsey Educational Publishers; 1999.

35. Hammer $\varnothing$, Harper DAT, Ryan PD. PAST: Paleontological statistics software package for education and data analysis. Palaeontologia Electronica 2001;4(1):9pp. http://palaeo-electronica.org/2001_1/past/issue1_01.htm. Consultado 12 May, 2018.

36. Traver BE, Williams MR, Fell RD. Comparison of within hive sampling and seasonal activity of Nosema ceranae in honey bee colonies. J Invertebr Pathol. 2012;(109):187193. 
37. Higes M, Nozal MJ, Álvaro A, Barrios L, Meana A, Martín-Hernández R, Bernal JL, Bernal J. The stability and effectiveness of fumagillin in controlling Nosema ceranae (Microsporidia) infection in honey bees (Apis mellifera) under laboratory and field conditions. Apidologie 2011;(42):364-377.

38. Williams GR, Sampson MA, Shutler D, Rogers REL. Does fumagillin control the recently detected invasive parasite Nosema ceranae in western honey bees (Apis mellifera)? J Invertebr Pathol 2008;(99):342-344.

39. Holt HL, Grozinger CM. Approaches and challenges to managing Nosema (Microspora: Nosematidae) parasites in honey bee (Hymenoptera: Apidae) colonies. J Econ Entomol 2016;(109):1487-1503.

40. Burnham AJ. Scientific advances in controlling Nosema ceranae (Microsporidia) infections in honey bees (Apis mellifera). Front Vet Sci 2019;(6):79.

41. van den Heever JP, Thompson TS, Otto SJG, Curtis, JM, Ibrahim A, Pernal SF. Evaluation of Fumagilin-B® and other potential alternative chemotherapies against Nosema ceranae-infected honeybees (Apis mellifera) in cage trial assays. Apidologie 2016;(47):617-630. 